Original article

\title{
Development and characterization of a novel fluorescent indicator protein PMCA4-GCaMP2 in cardiomyocytes
}

\author{
Tamer M. A. Mohamed ${ }^{\mathrm{a}, \mathrm{b}, 1}$, Riham Abou-Leisa ${ }^{\mathrm{a}, 1}$, Florence Baudoin ${ }^{\mathrm{a}}$, Nicholas Stafford ${ }^{\mathrm{a}}$, Ludwig Neyses ${ }^{\mathrm{a}, 2}$, \\ Elizabeth J. Cartwright ${ }^{\mathrm{a}, 2}$, Delvac Oceandy ${ }^{\mathrm{a}, *, 2}$ \\ a Institute of Cardiovascular Sciences, University of Manchester, Manchester Academic Health Sciences Centre, Manchester M13 9PT, UK \\ b Department of Biochemistry, Faculty of Pharmacy, Zagazig University, Zagazig, Egypt
}

\section{A R T I C L E I N F O}

Article history:

Received 5 February 2013

Received in revised form 4 July 2013

Accepted 11 July 2013

Available online 21 July 2013

\section{Keywords:}

PMCA4

GCaMP2

Local calcium

Cardiomyocytes

PMCA4 inhibitor

\begin{abstract}
A B S T R A C T
Isoform 4 of the plasma membrane calcium/calmodulin dependent ATPase (PMCA4) has recently emerged as an important regulator of several key pathophysiological processes in the heart, such as contractility and hypertrophy. However, direct monitoring of PMCA4 activity and assessment of calcium dynamics in its vicinity in cardiomyocytes are difficult due to the lack of molecular tools. In this study, we developed novel calcium fluorescent indicators by fusing the GCaMP2 calcium sensor to the N-terminus of PMCA4 to generate the PMCA4-GCaMP2 fusion molecule. We also identified a novel specific inhibitor of PMCA4, which might be useful for studying the role of this molecule in cardiomyocytes and other cell types.

Using an adenoviral system we successfully expressed PMCA4-GCaMP2 in both neonatal and adult rat cardiomyocytes. This fusion molecule was correctly targeted to the plasma membrane and co-localised with caveolin-3. It could monitor signal oscillations in electrically stimulated cardiomyocytes. The PMCA4-GCaMP2 generated a higher signal amplitude and faster signal decay rate compared to a mutant inactive PMCA4 ${ }^{\text {mut }} \mathrm{GCaMP2}$ fusion protein, in electrically stimulated neonatal and adult rat cardiomyocytes. A small molecule library screen enabled us to identify a novel selective inhibitor for PMCA4, which we found to reduce signal amplitude of PMCA4-GCaMP2 and prolong the time of signal decay (Tau) to a level comparable with the signal generated by PMCA $4{ }^{\text {mut }}$ GCaMP2. In addition, PMCA4-GCaMP2 but not the mutant form produced an enhanced signal in response to $\beta$-adrenergic stimulation. Together, the PMCA4-GCaMP2 and PMCA4 ${ }^{\text {mut }}$ GCaMP2 demonstrate calcium dynamics in the vicinity of the pump under active or inactive conditions, respectively.

In summary, the PMCA4-GCaMP2 together with the novel specific inhibitor provides new means with which to monitor calcium dynamics in the vicinity of a calcium transporter in cardiomyocytes and may become a useful tool to further study the biological functions of PMCA4. In addition, similar approaches could be useful for studying the activity of other calcium transporters during excitation-contraction coupling in the heart.
\end{abstract}

(c) 2013 Elsevier Ltd. All rights reserved.

\section{Introduction}

The plasma membrane calcium/calmodulin dependent ATPase (PMCA) is a ubiquitous calcium transporting enzyme which mainly functions to extrude intracellular calcium (reviewed in [1]). In nonexcitable cells PMCA is understood to play a major role in calcium extrusion [2], however in excitable cells (such as cardiomyocytes),

Abbreviations: PMCA, plasma membrane calcium/calmodulin dependent ATPase; NCX, sodium/calcium exchanger; SERCA, sarcoplasmic reticulum ATPase; nNOS, neuronal nitric oxide synthase; GECI, genetically encoded calcium indicator; GCaMP2, GFP-calmodulinbased GECI; NRCM, neonatal rat cardiomyocytes; ATA, aurintricarboxylic acid.

* Corresponding author at: Institute of Cardiovascular Sciences, University of Manchester, Manchester Academic Health Sciences Centre. Room 1.302 Stopford Bldg, Oxford Road, Manchester M13 9PT, UK. Tel.: + 44161 2751772; fax: + 441612755669.

E-mail address: Delvac.oceandy@manchester.ac.uk (D. Oceandy).

${ }^{1}$ Equally contributed to the work.

2 L.N., E.J.C. and D.O. are equal senior authors. its importance in respect to bulk calcium transport has traditionally been assumed to be minor. Using serial pharmacological inhibitors, several studies have shown that the majority of cytosolic calcium removal is via the sarcoplasmic reticulum ATPase (SERCA) and sodium/ calcium exchanger (NCX) (reviewed in [3]). PMCA1 and PMCA4 are the two isoforms expressed in the cardiomyocytes [4] and their high affinity for calcium has led to speculation that they may participate in, or fine tune, diastolic calcium extrusion [5,6]. Based on an alternative hypothesis, our group and others have identified a signalling role for isoform 4 of the PMCA (PMCA4). PMCA4 interacts with and modulates the activity of other molecules, which have significance in cardiac physiology. For example, PMCA4 modulates cardiac contractility and hypertrophy by regulation of neuronal nitric oxide synthase (nNOS) and calcineurin, respectively [7-10]. Both nNOS and calcineurin are calcium-dependent enzymes and it is therefore logical to speculate that PMCA4 may be important in defining calcium concentration within its vicinity and hence alter the activity of its interacting partners. 
Considering that PMCA4 might exert its signalling role by regulating local calcium concentration $[7,10,11]$, it is important to develop a novel tool to assess calcium dynamics in the vicinity of PMCA4. In this study we fused PMCA4 with a genetically encoded calcium indicator (GECI) $[12,13]$ and characterized its properties in situ in cardiomyocytes. We used the GFP-based GECI (GCaMP2), which exploits the calcium/calmodulin dependent rearrangement of recombinant GFP that results in an increase in fluorescence intensity upon binding with calcium/calmodulin [14]. It has been shown in a number of models that GCaMP2 fluorescent intensity is a function of calcium concentration [14]. It was also reported previously that using transgenic mouse technology, expression of GCaMP2 in the heart can be used to examine calcium transients in vivo [13]. The high brightness and stability of GCaMP2 enable the measurement of the rapid changes in $\mathrm{Ca}^{2+}$ transients in all regions of the beating mouse heart and for prolonged pacing and mapping studies in isolated, perfused hearts [13]. Here we used an adenoviral system to enable the delivery of GCaMP2 in isolated/cultured cardiomyocytes and assess the ability of this adenoviral-based GCaMP2 to detect rapid calcium fluctuations in isolated cardiomyocytes. We also generated adenoviruses to enable expression of two fusion molecules: PMCA4-GCaMP2 and PMCA4 ${ }^{\text {mut }}$ GCaMP2. In addition, through screening a medically optimised chemical library we have identified a new and highly selective PMCA4 inhibitor, which was very useful in studying the fusion fluorescent molecules. Our analyses suggest that the indicators were able to report calcium dynamics in the local vicinity of PMCA4 which was dependent on PMCA4 activity.

\section{Methods}

\subsection{Adenovirus}

Adenoviruses expressing the cytoplasmic GCaMP2 (cytoplasmic calcium sensor) [13] were generated by cloning GCaMP2 (a generous gift from J Nakai, Saitama, Japan) to the pAd/CMV/V5-DEST vector (Invitrogen) using the Gateway system (Invitrogen) following the manufacturer's instructions. To generate adenovirus expressing GCaMP2 linked to PMCA4 or PMCA4 ${ }^{\text {mut }}$, we cloned GCaMP2 cDNA to the $\mathrm{N}$ terminal end of PMCA4 or PMCA4 ${ }^{\text {mut }}$ and then subcloned the resulting construct to the $\mathrm{pAd} / \mathrm{CMV} / \mathrm{V} 5$-DEST vector as above.

\subsection{Isolation of adult and neonatal cardiomyocytes and western blot analysis}

Adult cardiomyocytes were isolated from 2 to 3-month old rats. In brief, rats were sacrificed by cervical dislocation, the hearts were rapidly removed and then perfused via the aorta with isolation solution pH 7.34 (134 mM NaCl; 11 mM Glucose; 4 mM KCl; 1.2 mM MgSO4; $1.2 \mathrm{mM} \mathrm{NaH2PO} 4$; $10 \mathrm{mM}$ HEPES) for $4 \mathrm{~min}$ followed by $9 \mathrm{~min}$ perfusion with a solution containing $0.6 \mathrm{mg} / \mathrm{ml}$ and $0.075 \mathrm{mg} / \mathrm{ml}$ of collagenase type II (Worthington) and proteases type XIV (Sigma-Aldrich), respectively. Hearts were then perfused with Tyrode solution containing $50 \mathrm{mM}$ taurine $\mathrm{pH} 7.34$ for $12 \mathrm{~min}$. The ventricles were cut from the heart and placed in Tyrode-taurine solution. The ventricles were then cut in half and pipetted up and down through a Pasteur pipette in $5 \mathrm{ml}$ of Tyrode-taurine solution to release the cardiomyocytes. The cardiomyocytes were then plated on laminin coated coverslips, infected with adenovirus and cultured for $48 \mathrm{~h}$ using a previously described protocol [15].

Neonatal rat cardiomyocytes (NRCM) were isolated from 1 to 3-day old Sprague Dawley rat neonates using a protocol as described previously [16]. Western blot analyses using anti-GFP (Abcam) and anti-GAPDH (Abcam) (loading control) were carried out as described before [17].

\subsection{Immunofluorescence}

Neonatal or adult rat cardiomyocytes were plated on laminin coated coverslips. Cells were washed twice with PBS and then fixed by adding $0.5 \mathrm{ml} \mathrm{4 \%} \mathrm{formaldehyde} \mathrm{for} 15 \mathrm{~min}$. Fixed cells were washed three times with PBS then permeabilised with $0.1 \%$ Triton X-100 for $15 \mathrm{~min}$ and blocked with $10 \%$ horse serum in PBS for $30 \mathrm{~min}$ at room temperature. Cells were then probed with monoclonal mouse FITC conjugated anti-GFP (Abcam) or monoclonal mouse anti-caveolin-3 antibodies (BD) $(1 / 100)$ diluted in $1 \%$ horse serum for $1.5 \mathrm{~h}$ at room temperature, washed three times in PBS and then labelled with TR conjugated antimouse $(1 / 200)$ (Jackson Lab) diluted in $1 \%$ horse serum for $1.5 \mathrm{~h}$ in the dark. The cells were then washed a further three times in PBS and once in water. Coverslips were mounted onto slides using a Prolong Antifade $^{\mathrm{TM}}$ Kit (Molecular Probes) and cell staining was visualized using a Leica SP5 confocal microscope.

\subsection{Calcium transient recordings using GCaMP2, PMCA4-GCaMP2 and PMCA4 ${ }^{\text {mut }}$ GCaMP2}

NRCM or adult rat cardiomyocytes were infected with adenovirus for the specific calcium sensor for $48 \mathrm{~h}$. In order to measure calcium transients the coverslips containing infected myocytes were placed in a bath with a cover slip base. This bath was positioned on the stage of an epifluorescence adapted inverted Olympus IX70 microscope fitted with an Olympus America camera. The myocytes were perfused with Tyrode solution and then field stimulated by two silver wire electrodes initiating an electrical current at a frequency of $1 \mathrm{~Hz}$. Calcium oscillations during myocyte contraction were recorded at $33^{\circ} \mathrm{C}$. Excitation was set at $485 \mathrm{~nm}$ using an ARC lamp (Cairn Inc), by means of a laser spot applied to small areas of the cell. The fluorescence emission at $525 \mathrm{~nm}$ was collected using a dichroic filter and a Cairn Integra photomultiplier tube. The data were collected and analyzed using Ionwizard software (Ionoptix Inc.). The calcium transients signal was represented as a ratio of the post-stimulation fluorescence, $\mathrm{F}$, divided by the mean pre-stimulation baseline fluorescence, $F_{0}$ for each cell. The single exponential tau was calculated using the Ionwizard software (Ionoptix Inc.) as the exponential decay time constant of the calcium transients following the exponential fitting of the calcium transients decay slope and calculated as tau $=1 / \mathrm{K}_{\text {fall }}$ where $\mathrm{K}_{\text {fall }}=$ exponential rate constant of the calcium decay. Caffeine experiments were carried out by perfusing the cardiomyocytes with normal Tyrode solution (TS). Then cells were rapidly perfused with $10 \mathrm{mM}$ caffeine for $3 \mathrm{~min}$ using an 8 channel local rapid perfusion system (MPER8 (Cairn research LTD)) which was placed in close proximity to the cell to ensure rapid application of caffeine. For $0 \mathrm{Na} / \mathrm{OCa}$ experiments the cells were perfused with $\mathrm{Na}^{+}$and $\mathrm{Ca}^{2+}$-free Tyrode solution (ONaOCa), in which all sodium salts were replaced with lithium salts, for $60 \mathrm{~s}$ prior to caffeine application. Indo-1 experiments were performed following methods described elsewhere [7,17].

\subsection{Microsomal preparation and coupled enzyme assay for PMCA4 activity}

HEK293 cells were infected with the PMCA4 [7], PMCA4-GCaMP2, PMCA4 ${ }^{\text {mut }}$ GCaMP2, GCaMP2, or LacZ adenovirus for $48 \mathrm{~h}$. Microsomal membrane preparations were carried out from the transfected cells as follows: cells were washed three times with PBS and then harvested in $5 \mathrm{ml}$ harvest solution $(1 \times \mathrm{PBS}, 0.26 \% 2 \mathrm{mg} / \mathrm{ml}$ aprotinin, $0.11 \%$ $2 \mathrm{mg} / \mathrm{ml}$ leupeptin, 0.1\% 0.1 M PMSF). Harvested cells were centrifuged at $1000 \times \mathrm{g}$ for $10 \mathrm{~min}$ at $4{ }^{\circ} \mathrm{C} .3 \mathrm{ml}$ hypotonic solution $(10 \mathrm{mM}$ Tris-HCl, pH 7.5, 1 mM MgCl2, 0.5 mM EGTA, 2 mM DTT, 0.2\% 2 mg/ml aprotinin, and $0.05 \% 2 \mathrm{mg} / \mathrm{ml}$ leupeptin) was added to the cell pellet for 10 min on ice. Swelled cells were homogenised in a Dounce homogenizer before addition of $3 \mathrm{ml}$ homogenate solution ( $10 \mathrm{mM}$ Tris- $\mathrm{HCl}$, pH 7.5, $2 \mathrm{mM}$ DTT, $0.38 \mathrm{M}$ sucrose, $0.3 \mathrm{M} \mathrm{KCl}, 0.2 \% 2 \mathrm{mg} / \mathrm{ml}$ aprotinin, and $0.05 \% 2 \mathrm{mg} / \mathrm{ml}$ leupeptin); homogenization was completed to seal the 
vesicles. The cell homogenate was centrifuged at $1500 \times \mathrm{g}$ for $20 \mathrm{~min}$ to remove the cellular debris. $60 \mu \mathrm{l} 0.25 \mathrm{mM}$ EDTA and $1.08 \mathrm{ml}$ of $2.5 \mathrm{M}$ $\mathrm{KCl}$ were added to the supernatant and centrifuged at $100,000 \times \mathrm{g}$ for $40 \mathrm{~min}$ at $4{ }^{\circ} \mathrm{C}$. The resulting pellet was re-suspended in $0.4 \mathrm{ml}$ final solution (10 mM Tris-HCl, pH 7.5, $1 \mathrm{mM}$ DTT, $0.19 \mathrm{M}$ sucrose, $0.15 \mathrm{M} \mathrm{KCl}$, $0.2 \% 2 \mathrm{mg} / \mathrm{ml}$ aprotinin, $0.05 \% 2 \mathrm{mg} / \mathrm{ml}$ leupeptin and $0.02 \mathrm{mM} \mathrm{CaCl}$ ). The coupled enzyme assay was carried out as previously described [18].

\subsection{Global intracellular calcium assay using GCaMP2 fusion proteins}

To compare the basal fluorescence signal intensity between PMCA4GCaMP2 and PMCA4 ${ }^{\text {mut }}$ GCaMP2 we used a modification of the method described in [19]. Briefly, NRCM were plated in black walled glass bottom 96 well plates and infected with PMCA4-GCaMP2 or PMCA $4{ }^{\text {mut }}$ GCaMP2 for $48 \mathrm{~h}$. On the day of the assay, the medium was replaced by $100 \mu \mathrm{l}$ of fresh Hanks' balanced salt solution (HBSS)bovine serum albumin (BSA)-probenecid solution (HBSS, 1\%w/v BSA and $2.5 \mathrm{mM}$ probenecid) in which NRCM were incubated for $30 \mathrm{~min}$ at $37^{\circ} \mathrm{C}$. Cells were then washed with $100 \mu \mathrm{l}$ of HBSS-BSA-probenecid and finally maintained in $80 \mu \mathrm{l}$ of fresh HBSS-BSA-probenecid containing $1.26 \mathrm{mM}$ calcium chloride. Baseline fluorescence $(\mathrm{F})$ was measured for three minutes with filters for excitation at $485 \mathrm{~nm}$ and for emission at $538 \mathrm{~nm}$ using a BMG FLUOstar Omega plate reader. Following baseline fluorescence measurement, $14 \mu$ of $\mathrm{F}_{\min }$ solution (HBSS-BSA probenecid, $\mathrm{pH}$ 7.45, $100 \mu \mathrm{M}$ ionomycin, $10 \mu \mathrm{M}$ Thapsigargin and $20 \mathrm{mM}$ EGTA, $\mathrm{pH}$ 8.0) was added to each well to deplete the calcium from the cells and leave only the autofluorescence which was measured for $120 \mathrm{~min}\left(\mathrm{~F}_{\min }\right)$. Finally, $16 \mu \mathrm{l}$ of $\mathrm{F}_{\max }(250 \mathrm{mM}$ calcium chloride in HBSS-BSA probenecid) solution was added to saturate the cells with calcium and fluorescence was measured for another five minutes $\left(\mathrm{F}_{\max }\right)$. To test the effect of various calcium concentrations on the fluorescent intensity of the sensors, we varied the calcium chloride concentration in the $\mathrm{F}_{\max }$ solution to produce corresponding free calcium concentration in the medium ranging from $100 \mathrm{nM}$ to $10 \mathrm{mM}$ as calculated using an online software tool based on the Fabiato formula (http://www.stanford. edu/ cpatton/CaEGTA-NIST.htm).

\subsection{Identification of a novel PMCA4 inhibitor}

Using a modified coupled enzyme ATPase assay for PMCA4 activity [18], we screened a library of 1280 medically optimised drug-like molecules (LOPAC ${ }^{1280}$ ) (Sigma-Aldrich) against PMCA4 activity in membrane microsomes derived from HEK293 cells overexpressing PMCA4 protein as described in detail above under Section 2.5. The first round of screening was used to select compounds with an inhibitory effect of more than $50 \%$ at $10 \mu \mathrm{M}$ concentration. These preselected compounds underwent a counter assay to identify whether they exhibited an effect on the coupled enzyme assay components where $10 \mathrm{mM}$ adenosine $5^{\prime}$-diphosphate sodium salt (ADP) was used to start up the coupled enzyme reaction instead of microsomes. Identified inhibitors which had no effect on the coupled enzyme assay components were then confirmed with a fresh solid bought from Sigma-Aldrich and dose response curves for these compounds were carried out. To test the specificity of the most potent identified inhibitor for PMCA4 over other ATPases expressed in the heart, we used membrane microsomes derived from HEK293 cells overexpressing PMCA4, SERCA2a or PMCA1 $[7,17]$ and purified $\mathrm{Na}^{+} / \mathrm{K}^{+}$ATPase (Sigma-Aldrich). The inhibitory effect was tested over a range of concentrations in each microsome using the modified coupled enzyme assay [18].

\subsection{Statistical analysis}

Data are expressed as mean \pm SEM. Student's $t$ test or one way ANOVA followed by post-hoc multiple comparison were used where appropriate. The probability level for statistical significance was set at $\mathrm{p}<0.05$

\section{Results}

3.1. Adenoviral-based expression of GCaMP2 in neonatal and adult rat cardiomyocytes

We generated adenovirus expressing the GCaMP2 molecule driven by a cytomegalovirus (CMV) promoter. The virus successfully induced the expression of GCaMP2 in cultured neonatal rat cardiomyocytes and adult rat cardiomyocytes. Using confocal immunofluorescence analysis we detected that GCaMP2 was mainly localised in the cytoplasmic compartment of both types of cells (Fig. 1A). Under electrical stimulation $(1 \mathrm{~Hz})$ both neonatal and adult cardiomyocytes overexpressing GCaMP2 showed consistent signal oscillations (Fig. 1B). Furthermore, stimulation of adult cardiomyocytes with $100 \mathrm{nM}$ isoprenaline resulted in a significant enhancement in the GCaMP2 signal (Fig. 1C). These results showed that our GCaMP2 expressing adenovirus was able to monitor calcium oscillations in cultured neonatal and adult rat cardiomyocytes during the excitation-contraction coupling process.

\subsection{Generation of PMCA4-GCaMP2 fusion proteins}

To generate a PMCA4 based GCaMP2 indicator, we cloned GCaMP2 directly to the N-terminus of full-length PMCA4 (PMCA4-GCaMP2).

A
Adult Rat Cardiomyocytes + Ad-GCaMP2

Ad
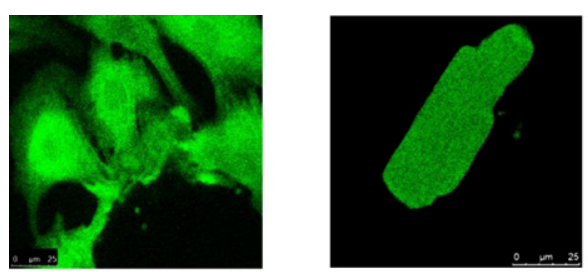

B

Neonatal Rat Cardiomyocytes Adult Rat Cardiomyocytes
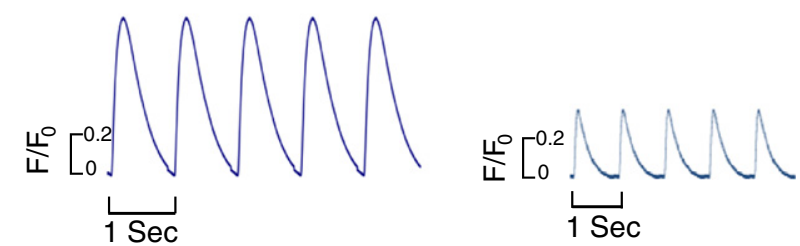

C

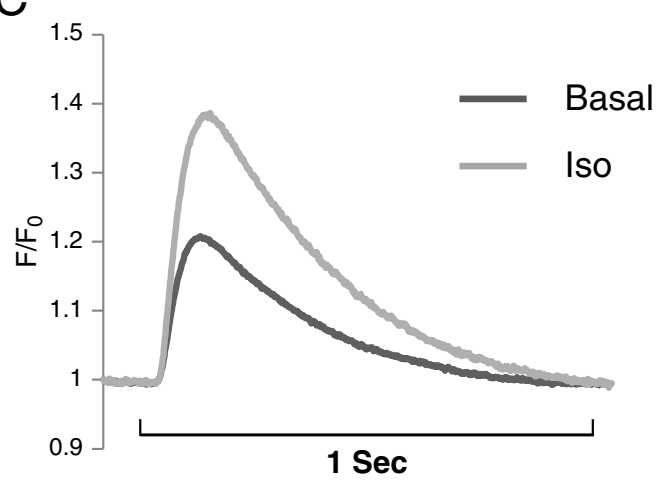

Fig. 1. Adenoviral expression of GCaMP2 in isolated cardiomyocytes. (A) Neonatal and adult rat cardiomyocytes were infected with adenovirus expressing GCaMP2 calcium sensor (Ad-GCaMP2). Expression and localization of GCaMP2 was detected using anti-GFP antibody. (B) Representative signal oscillations produced by the GCaMP2 sensor in neonatal and adult rat cardiomyocytes following electrical stimulation at $1 \mathrm{~Hz}$. (C) Effect of $100 \mathrm{nM}$ isoprenaline on calcium oscillations detected by GCaMP2 sensor in adult cardiomyocytes. 
We also fused GCaMP2 to an inactive mutant form of PMCA4, which carries an $\mathrm{Asp}^{672} \mathrm{Glu}$ amino acid substitution, thereby reducing the ATPase activity by $90 \%$ [20] (we named the recombinant molecule as
PMCA4 ${ }^{\text {mut }}$ GCaMP2) (Fig. 2A). We then produced adenoviruses to enable efficient gene delivery and expression in isolated neonatal and adult rat cardiomyocytes. Expressions of both molecules were CMV
A

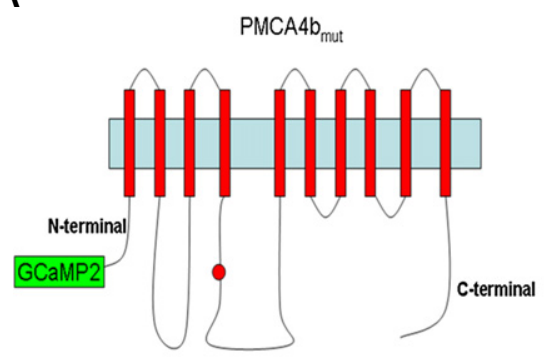

B

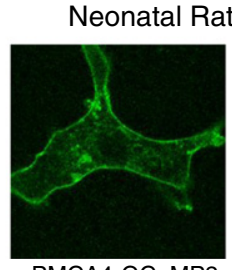

+ PMCA4-GCaMP2

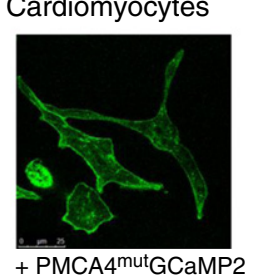

$\mathrm{D}$

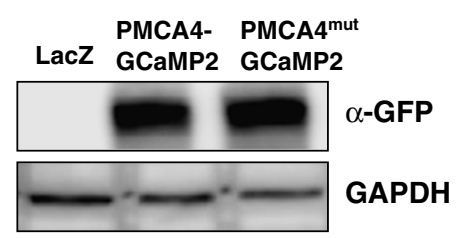

PMCA4b

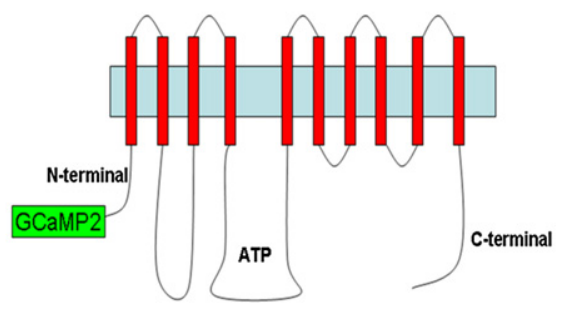

C Adult Rat Cardiomyocytes
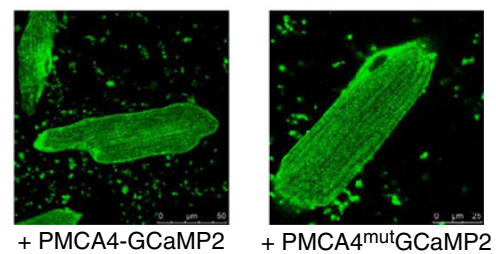

$E$

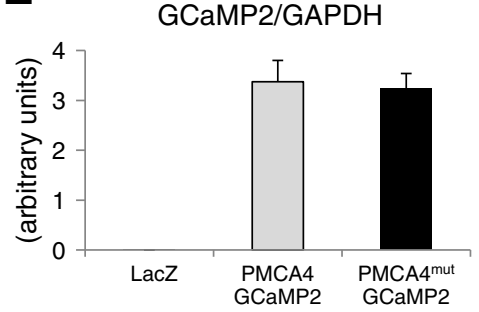

$\mathrm{F}$

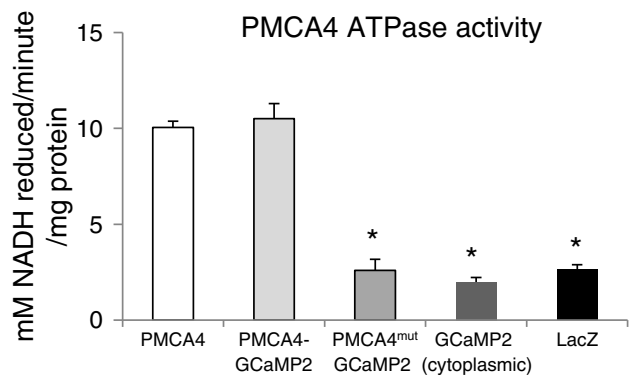

G

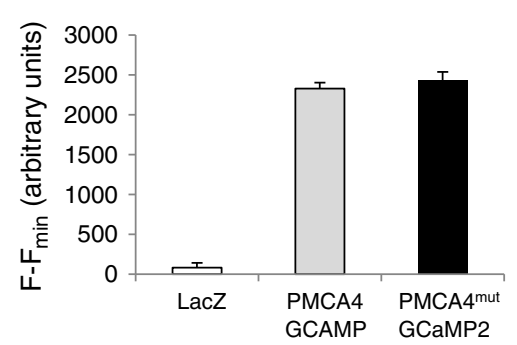

$\mathrm{H}$

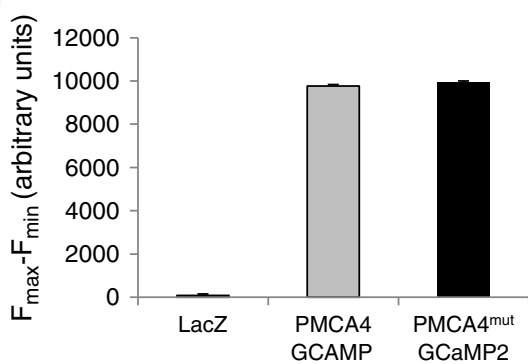

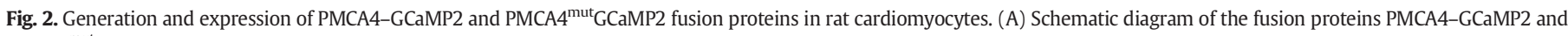

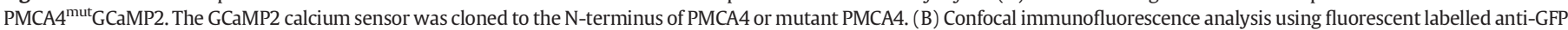

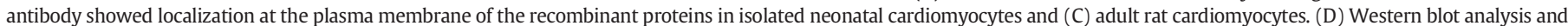

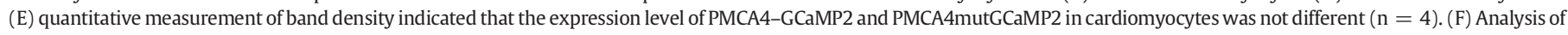

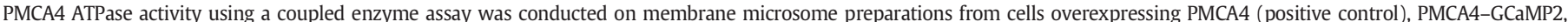

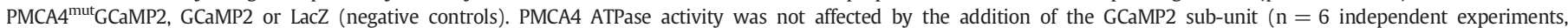

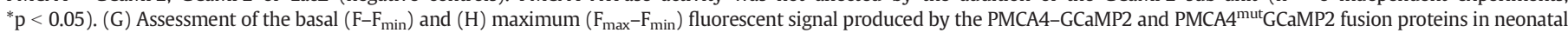
rat cardiomyocytes showed a non-significant difference in fluorescence intensity under either condition ( $\mathrm{n}=24$ wells in each group from 3 independent experiments). 
A

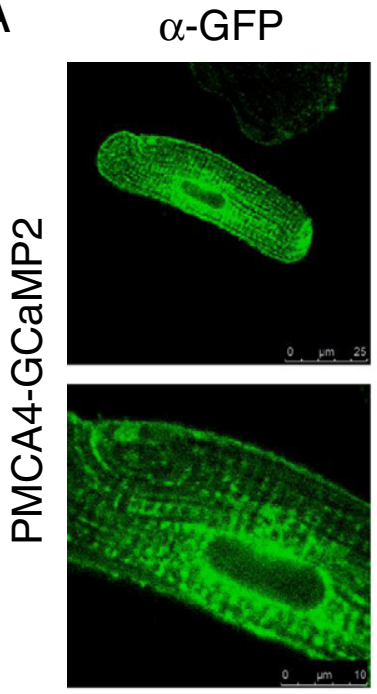

B

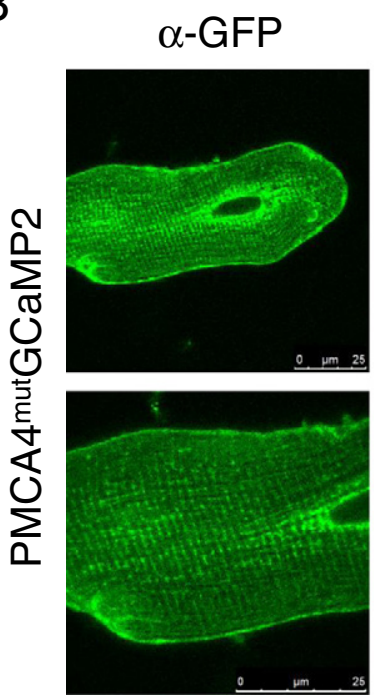

$\alpha-$ Cav3
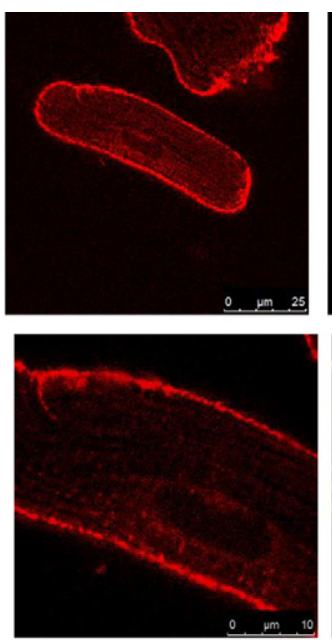

$\alpha-$-Cav3
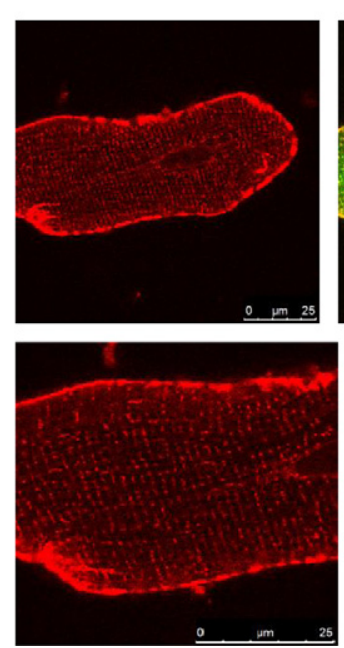

merge
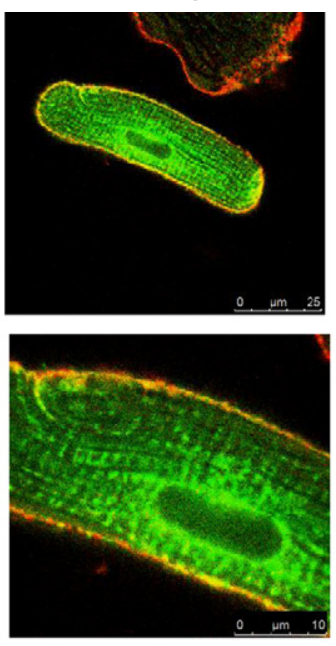

merge
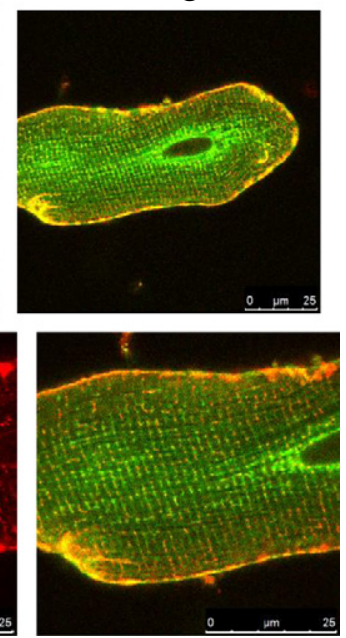

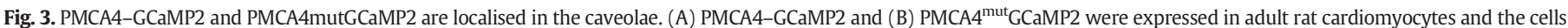

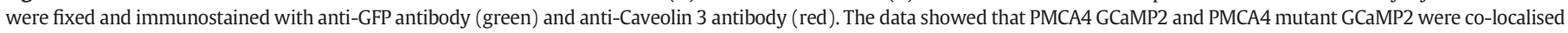
with caveolin 3.

promoter-driven. To determine the localization of these recombinant proteins in transduced cardiomyocytes we used confocal immunofluorescence analysis using anti-GFP antibodies. Results presented in Fig. 2B\&C showed the expected sub-cellular localization of both molecules, i.e. in the plasma membrane. We performed Western blot analysis to ascertain whether introduction of the Asp ${ }^{672}$ Glu mutation had affected protein expression levels (Fig. 2D). Quantitative band density analysis indicated that the level of protein expression was comparable between PMCA4-GCaMP2, PMCA4 ${ }^{\text {mut }}$ GCaMP2 and GCaMP2 sensors (Fig. 2E).

To assess whether addition of GCaMP2 to the PMCA4 affected its enzymatic activity we conducted an in vitro ATPase assay using a coupled enzyme assay as described previously [18]. As shown in Fig. 2F there were no significant effects of GCaMP2 addition on the ATPase activity of PMCA4. As expected, the PMCA4 ${ }^{\text {mut }}$ GCaMP2 showed significantly lower ATPase activity which was comparable to levels shown by the negative controls (cells expressing cytoplasmic GCaMP2 or LacZ).

It was also important to evaluate whether the introduction of the Asp ${ }^{672}$ Glu mutation in the PMCA4 subunit affected the basal and maximum fluorescence signal intensities of the GCaMP2 subunit. To address this question we conducted experiments to assess the basal fluorescence (F) in NRCM expressing either PMCA4-GCaMP2 or PMCA $4{ }^{\text {mut }}$ GCaMP2 protein. We then added $F_{\text {min }}$ solution (Ionomycin to release the calcium from the stores and make the membrane permeable to calcium, thapsigargin to block the SR calcium reuptake and EGTA to chelate the calcium from the cells) to deplete the intracellular calcium from the cells and record only the fluorescence of the $\mathrm{Ca}^{2+}$-free fusion proteins $\left(F_{\min }\right)$ in the cells. This determination is possible because the cellular autofluroescence independent of the fusion proteins was negligible (see LacZ controls). This was followed by addition of $\mathrm{F}_{\max }$ solution containing $250 \mathrm{mM}$ calcium chloride (Fig. 2G-H) or $\mathrm{F}_{\max }$ solution containing variable calcium chloride concentrations in the presence of ionomycin/thapsigargin (from the $F_{\text {min }}$ solution) to introduce the desired free calcium levels ( $100 \mathrm{nM}-10 \mathrm{mM}$; Supplementary Fig. 1). As shown in Fig. 2G-H and Supplementary Fig. 1, PMCA4-

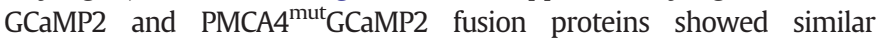
fluorescence intensity under different conditions, suggesting that the 
introduction of the point mutation did not alter the fluorescence intensity of the sensor.

\subsection{PMCA4-GCaMP2 and PMCA4 ${ }^{m u t} G C a M P 2$ are localised in caveolae}

We and others have suggested that in cardiomyocytes PMCA4 is localised in caveolae $[21,22]$. To analyse whether the recombinant PMCA4-GCaMP2 and PMCA4 ${ }^{\text {mut }}$ GCaMP2 were also targeted to the same sub-cellular compartment we conducted confocal immunoflorescence imaging using anti-GFP antibodies as well as anti-Cav3 antibodies. Fig. 3A\&B clearly show the co-localisation of PMCA4-GCaMP2 and PMCA4 ${ }^{\text {mut }}$ GCaMP2 with caveolin-3 (marker for caveolae) in adult rat cardiomyocytes. Taken together, the data suggested that our novel PMCA based GCaMP2 indicator was enzymatically active and correctly targeted to the caveolae of isolated cardiomyocytes.

\subsection{PMCA4-GCaMP2 exhibited stronger calcium oscillation signals} compared to PMCA4 ${ }^{\text {mut }}$ GCaMP2

To assess the calcium oscillations detected by PMCA4-GCaMP2 and PMCA $4{ }^{\text {mut }}$ GCaMP2 during excitation-contraction coupling, we used neonatal rat cardiomyocytes overexpressing these fusion proteins and electrically stimulated them at $1 \mathrm{~Hz}$. The results of these experiments showed that the signal amplitude produced by PMCA ${ }^{\text {mut }}$ GCaMP2 comprised only $50 \%$ of that generated by PMCA4-GCaMP2 (Fig. 4A\&B). Furthermore, analysis of the rate of signal decay confirmed that the active PMCA4 exhibited a significantly faster decay rate compared to the PMCA4 mutant fusion protein (Fig. 4C). Since PMCA4 ${ }^{\text {mut }}$ GCaMP2 displayed almost negligible enzymatic activity (Fig. 2F) the signal produced by this molecule likely reflected calcium changes in its local vicinity independent of PMCA4 activity. The difference in the signal amplitude and decay rate between the mutant and non-mutant molecule might be caused by the combination of PMCA4 activity and the change in local calcium concentration.
3.5. PMCA4-GCaMP2 fusion protein can detect PMCA4 involvement in calcium extrusion at the plasma membrane

Next we conducted experiments to override the sarcoplasmic reticulum (SR) filling by stimulation with $10 \mathrm{mM}$ caffeine. This will open the ryanodine receptors (RYR), which in turn will elevate the cytoplasmic calcium. In this situation the only mechanism for calcium removal will be the plasma membrane calcium extrusion systems, mainly mediated by the sodium calcium exchanger (NCX) and PMCA. The results of these experiments showed that the calcium signal produced by PMCA4 ${ }^{\text {mut }}$ GCaMP2 was $80 \%$ lower in amplitude compared to the other two sensors (Fig. 5A\&B). However, the calcium signal detected by the PMCA4-GCaMP2 protein was similar to that detected by the cytoplasmic GCaMP2 (Fig. 5A). Whilst we would expect a large increase in the signal amplitude detected by PMCA4-GCaMP2 under these conditions due to the exclusive removal of intracellular calcium through the plasma membrane systems, for the signal to rise to the level detected by cytoplasmic GCaMP2 was not expected. A likely explanation for this is saturation of the GCaMP2 sensor with high levels of calcium following caffeine application [3]. It was previously reported that the GCaMP2 sensor has the advantage of a low Kd value $(\sim 150 \mathrm{nM})$ which enables the detection of relatively small changes in calcium, such as those occurring in cardiomyocytes under physiological conditions and during excitation contraction coupling; however, its detection ability for changes in calcium concentration was limited to $\sim 1 \mu \mathrm{M}$ free calcium at which point the sensor signal becomes saturated $[14,23,24]$.

We conducted further experiments in the presence of an external solution of $0 \mathrm{Na}^{+} / 0 \mathrm{Ca}^{2+}$ to inhibit NCX activity and force the cell to extrude calcium only through PMCAs. As shown in Fig. 5C the rate of calcium decay detected by PMCA4-GCaMP2 was faster compared to that detected by cytoplasmic GCaMP2. Interestingly, PMCA $4{ }^{\text {mut }}$ GCaMP2 showed a lower amplitude and slower signal decay rate compared to PMCA4-GCaMP2 (Fig. 5D). The calcium decay rate as indicated by the time to $50 \%$ decay to baseline was significantly faster for the fluorescent signal recorded by PMCA4-GCaMP2 sensor compared to the other
A

PMCA4-GCaMP2

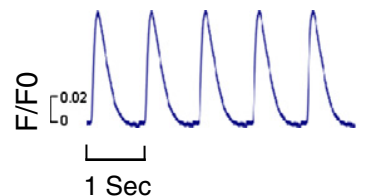

B

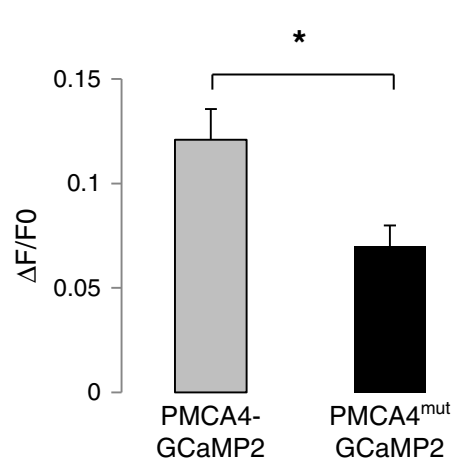

PMCA4mutGCaMP2

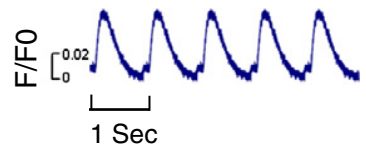

C

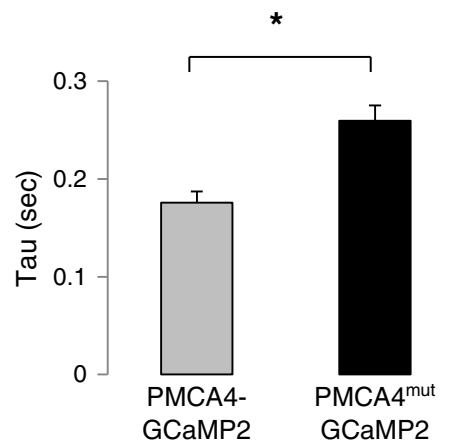

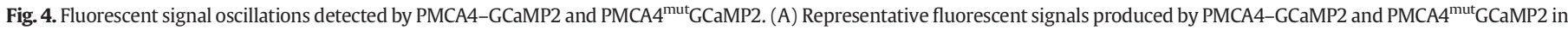

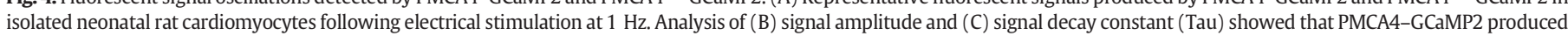
higher signal amplitude and faster decay rate compared to PMCA $4{ }^{\text {mut }} \mathrm{GCaMP} 2$ ( $\mathrm{n}=12-14$ cells in each group from 3 independent experiments, ${ }^{*} \mathrm{P}<0.05$ ). 
A

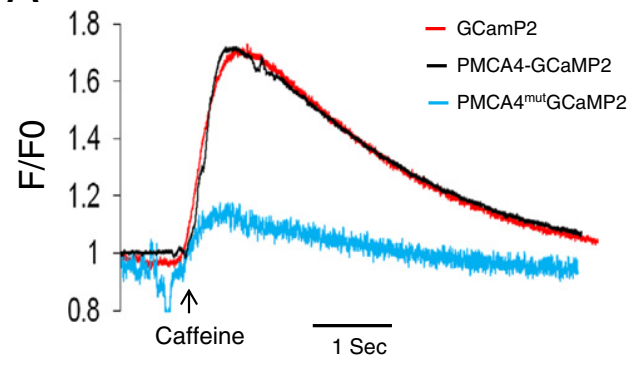

C

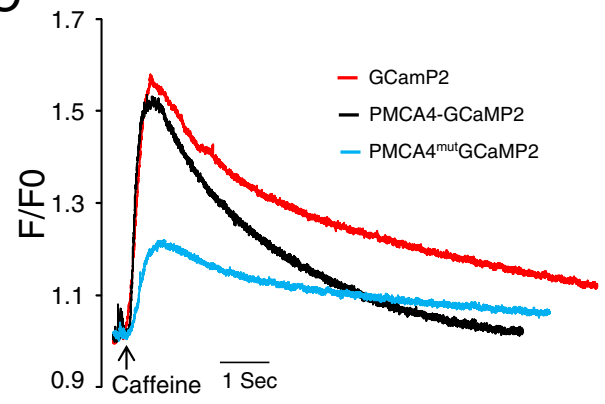

B

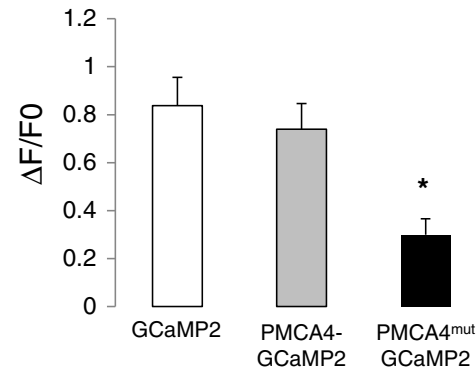

D

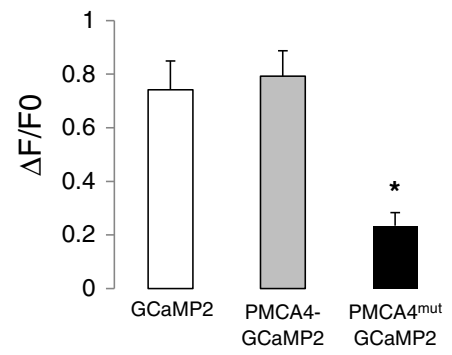

$E$

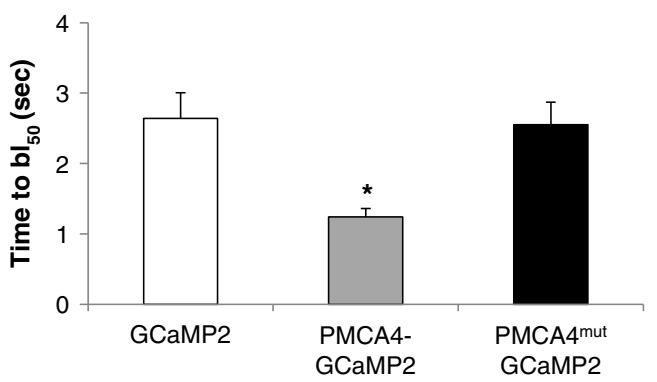

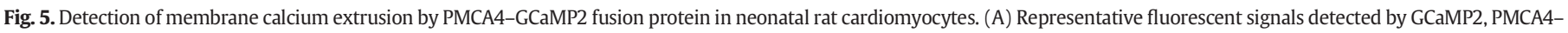

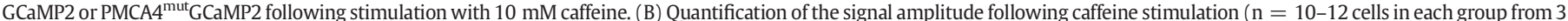

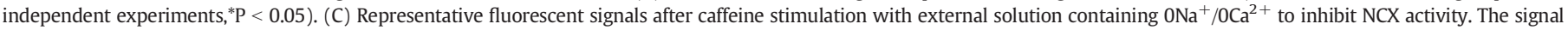

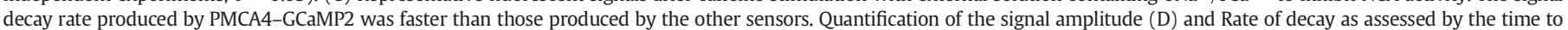

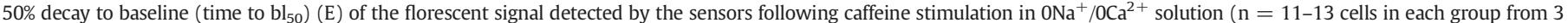
independent experiments, $\left.{ }^{*} \mathrm{P}<0.05\right)$.

sensors (Fig. 5E). Our data indicated that the PMCA4-GCaMP2 fusion protein was an active molecule, in particular if other means of calcium extrusion were inhibited.

\subsection{Identification of a novel PMCA4 inhibitor}

To further analyse the properties of the PMCA4-GCaMP2 molecule, experiments using a specific PMCA4 inhibitor were required. Since a specific and potent inhibitor of PMCA4 was not available [11] we screened a library of medically optimised drug-like molecules to identify novel PMCA4 inhibitors using a modified colorimetric ATPase assay [18]. We successfully identified eleven compounds that inhibited PMCA4 activity with an $\mathrm{IC}_{50}$ ranging from $0.1 \mu \mathrm{M}$ to $6 \mu \mathrm{M}$ (Supplementary Figs. 1\&2). The compound with the highest inhibitory effect was aurintricarboxylic acid (ATA), a polyaromatic carboxylic acid derivative. In vitro, the effect of ATA was dose-dependent and specific. At a concentration of 150 nM ATA inhibited PMCA4 activity by $50 \%$ and blocked almost all of the PMCA4 activity at a concentration of $1 \mu \mathrm{M}$ (Fig. 6A\&B). We then tested the specificity of ATA against other major ATPases (SERCA2a and $\mathrm{Na}^{+} / \mathrm{K}^{+}$ATPase) and the other PMCA isoform expressed in the heart (PMCA1) using the same coupled enzyme assay used in the screening [18]. ATA did not inhibit the $\mathrm{Na}^{+} / \mathrm{K}^{+}$ATPase, which is a related P-type ATPase and only produced a minor effect on the second isoform of PMCA expressed in the heart (PMCA1) as well as SERCA2a (Fig. 6B). Furthermore, ATA at a dose as high as $10 \mu \mathrm{M}$ did not alter the global calcium transient as recorded in adult cardiomyocytes using the ratio-metric dye Indo-1 (Fig. 6C-E), an aggregate measure of the activities of calcium transporters including SERCA, the Ryanodine receptor and the $\mathrm{Na}^{+} / \mathrm{Ca}^{2+}$ exchanger. This is consistent with the idea that PMCA4 is not involved in the regulation of bulk contractile calcium $[3,17]$.

\subsection{Effects of PMCA4 inhibition on the PMCA4-GCaMP2 fusion proteins}

To ensure that ATA did not directly affect the fluorescent intensity of the GCaMP2 sensor we analyzed fluorescent signals produced by NRCM expressing cytoplasmic GCaMP2 in the presence of $10 \mu \mathrm{M}$ ATA. As shown in Fig. 7A ATA treatment did not alter the signal amplitude nor decay rate of the cytoplasmic GCaMP2 sensor. We then performed experiments to investigate the effect of PMCA4 inhibition on the fluorescent signals produced by the PMCA4-GCaMP2 and PMCA4 ${ }^{\text {mut }}$ GCaMP2 
A

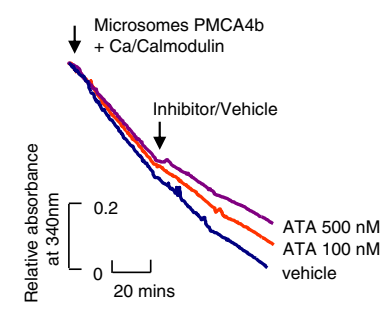

C

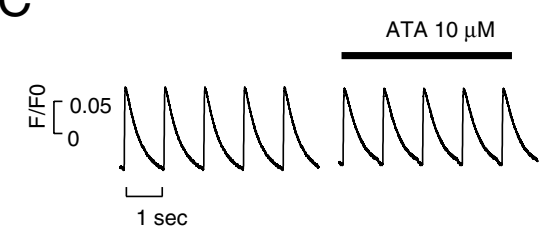

B

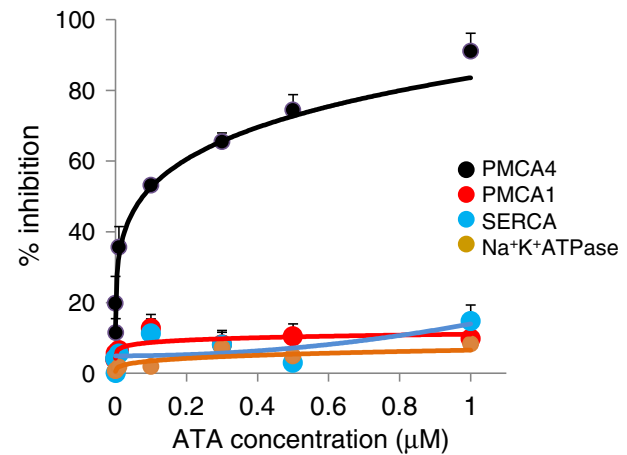

D

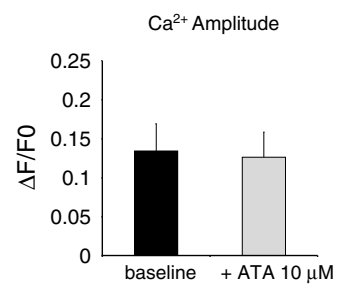

$E$

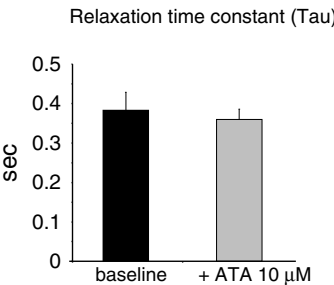

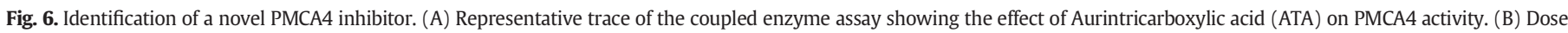

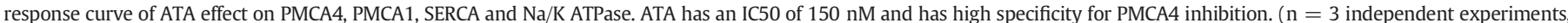

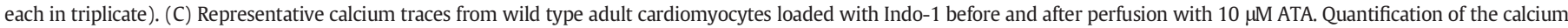

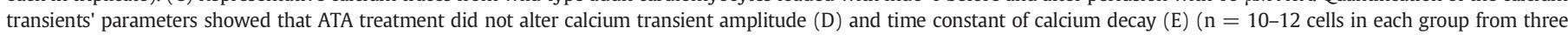
animals).

molecules in isolated cardiomyocytes. ATA significantly reduced the signal amplitude by over $45 \%$ and prolonged the time of signal decay (Tau) of the PMCA4-GCaMP2 molecule to a comparable level to the signal generated by PMCA $4{ }^{\text {mut }} \mathrm{GCaMP} 2$. In contrast, signal amplitude as well as signal decay produced by the PMCA4 ${ }^{\text {mut }}$ GCaMP2 remained unaffected following ATA treatment (Fig. 7B\&C). In addition, perfusion of $10 \mu \mathrm{M}$ ATA for 5 min prior to caffeine application did not change the caffeine-induced signal of the cytoplasmic GCaMP2 and PMCA $4{ }^{\text {mut }}$ GCaMP2. In contrast, ATA significantly reduced the caffeineinduced signal amplitude of the PMCA4-GCaMP2 to the level comparable to that produced by the PMCA4 ${ }^{\text {mut }}$ GCaMP2 (Fig. 7D). Together, our data confirms that the signal produced by the PMCA $4{ }^{\text {mut }}$ GCAMP2 indicates local calcium levels independent of the PMCA4 activity. The difference in the amplitude between the two sensors may be attributable to the activity of PMCA4.

3.8. PMCA4 is highly activated following $\beta$-adrenergic signalling in adult cardiomyocytes

It was previously reported that PMCA4 is also activated by protein kinase $A$ and $C$ (PKA, PKC) dependent processes [25]. PKA phosphorylates PMCA4 at a serine residue which is located downstream of the calmodulin binding domain, thus this activation may be due to the modulation of the autoinhibitory domain [26]. Protein kinase A is a cAMP-dependent enzyme and is activated by $\beta$-adrenergic stimulation [27]. We therefore tested whether PMCA4-GCaMP2 was activated following $\beta$-adrenergic stimulation. To address this question we conducted experiments on adult rat cardiomyocytes overexpressing either PMCA4-GCaMP2 or PMCA4 ${ }^{\text {mut } G C a M P 2 . ~ T h e s e ~ c e l l s ~ w e r e ~}$ electrically stimulated at $1 \mathrm{~Hz}$ and the calcium oscillations were recorded for each cell before and after stimulation with $100 \mathrm{nM}$ isoproterenol (Fig. 8A-B). The results of these experiments showed that PMCA4-GCaMP2 fusion protein detected a twofold elevation in the calcium oscillation amplitude and a significantly faster decay rate. However, no increase in signal amplitude or decay rate was detected by PMCA4 ${ }^{\text {mut }} \mathrm{GCaMP2}$ (Fig. $8 \mathrm{C}-\mathrm{D}$ ). These results showed that PMCA4-GCaMP2 but not PMCA4 ${ }^{\text {mut }}$ GCaMP2 was activated following $\beta$-adrenergic stimulation in adult cardiomyocytes.

\section{Discussion}

In this study we have generated novel fusion proteins PMCA4GCaMP2 and PMCA4 ${ }^{\text {mut }}$ GCaMP2 that can be efficiently delivered to isolated/cultured cardiomyocytes by using an adenoviral system. Both molecules are localised in the caveolae, which is in agreement with previous reports on sub-cellular localization of PMCA4 [22]. The key findings from the present study are: i) Both PMCA4-GCaMP2 and PMCA4 ${ }^{\text {mut }}$ GCaMP2 report $\mathrm{Ca}^{2+}$ transients; however, the signal amplitude of the mutant molecules was 50\% less than the wild type one. Given that the enzymatic activity of the mutant protein was almost negligible, the signal reported by the PMCA $4{ }^{\text {mut }}$ GCaMP2 might represent local calcium fluctuation in the vicinity of the molecule independent of the pump activity, whereas the signal reported by the PMCA4-GCaMP2 might be caused by the combination of PMCA4 activity and the change in local calcium. ii) Using a compound library screen we have identified aurintricarboxylic acid (ATA) as a novel specific PMCA4 inhibitor. iii) Inhibition of PMCA4 activity by ATA modified signal amplitude and decay rate of PMCA4-GCaMP2 to a 
level similar with that of PMCA $4{ }^{\text {mut }}$ GCaMP2. This implies that the calcium pumping activity of PMCA4 might change the calcium flux in and around the pump.

One may argue that the lower signal produced by PMCA $4{ }^{\text {mut }} \mathrm{GCaMP} 2$ might be caused by a direct effect of the genetic mutation within PMCA4 upon the GCaMP2 subunit, e.g. by causing protein misfolding and reducing protein level or signal intensity. Our data showed that the introduction of $\mathrm{Asp}^{672} \mathrm{Glu}$ did not affect protein expression level and localization. In addition, measurement of signals under basal and calcium saturated conditions revealed that both PMCA4-GCaMP2 and

A

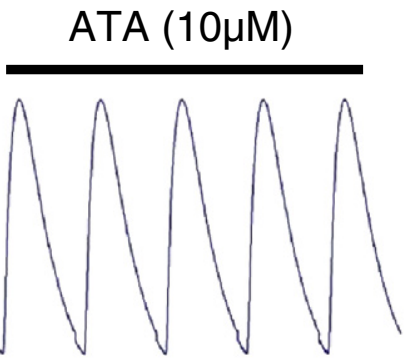

Change in amplitude

Relaxation time constant
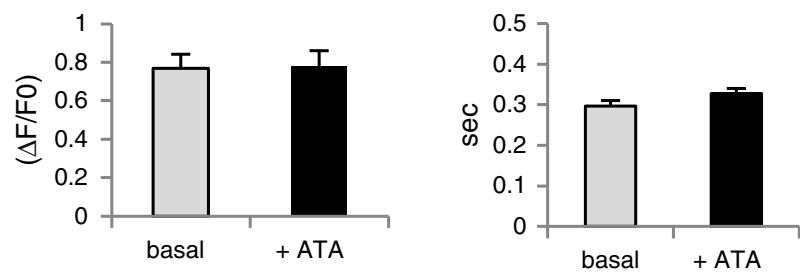

B
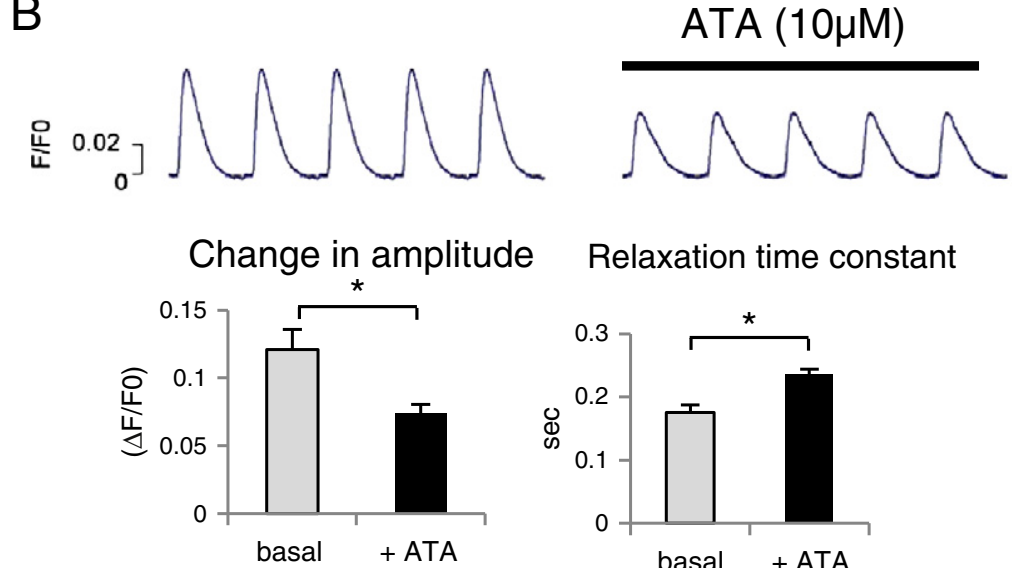

Relaxation time constant

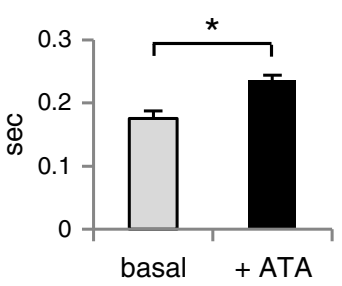

C
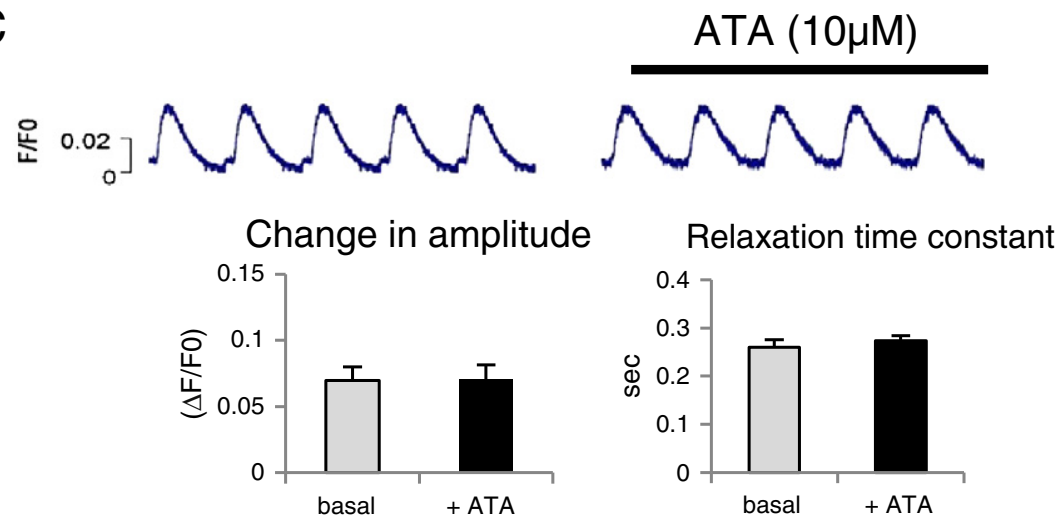

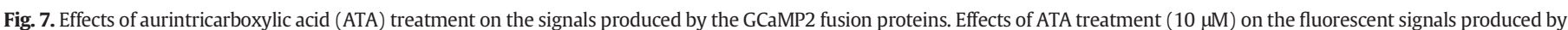

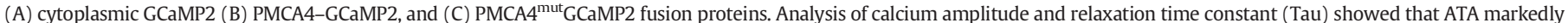

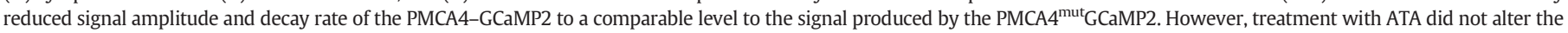

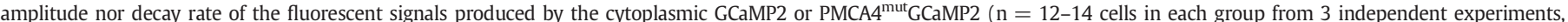

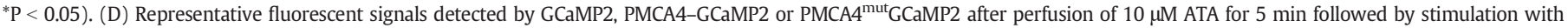

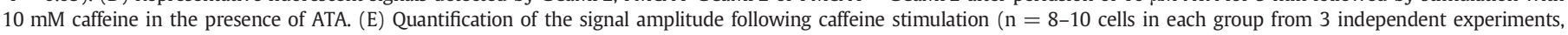
$\left.{ }^{*} \mathrm{P}<0.05\right)$. 


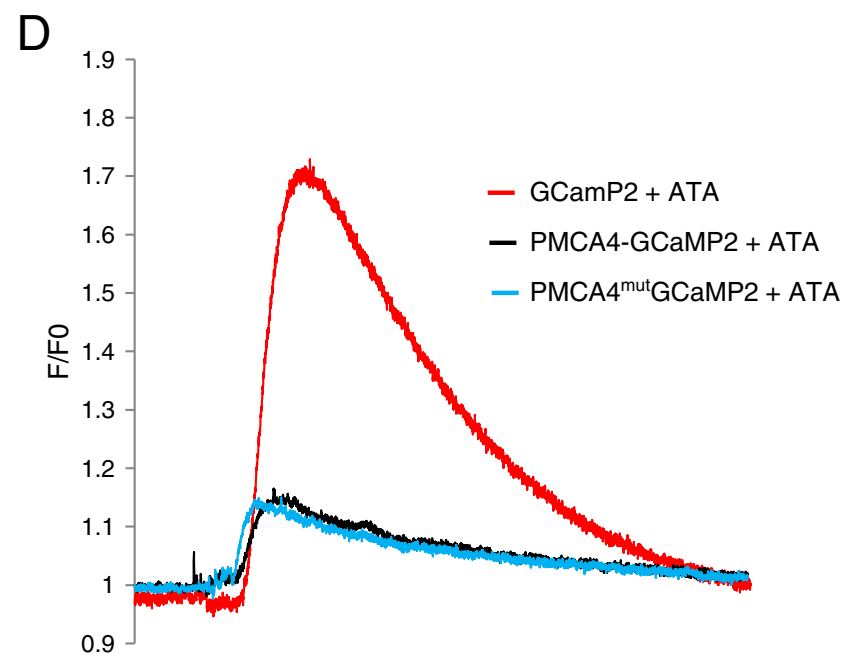

$\mathrm{E}$

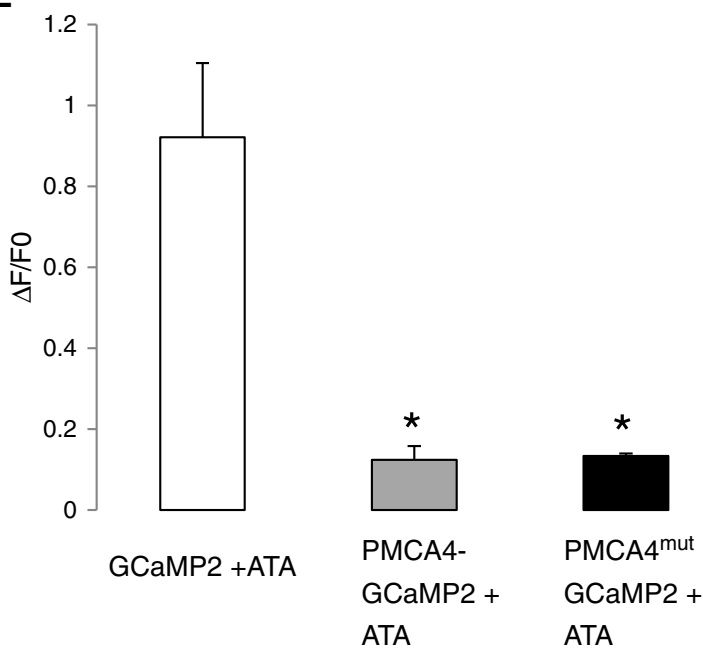

Fig. 7 (continued).

PMCA $4{ }^{\text {mut }}$ GCaMP2 fusion proteins produced similar levels of fluorescent intensity, suggesting that the GCaMP2 subunit in both fusion molecules displayed similar efficiency in detecting calcium. Therefore, the difference in the signals produced by PMCA4-GCaMP2 and PMCA $4{ }^{\text {mut }}$ GCaMP2 following electrical stimulation or after caffeine application was likely due to the difference of calcium levels around the pump, which might be caused by the activity of PMCA4. This has been further confirmed by our finding that treatment with a novel PMCA4 inhibitor (ATA) reduced the signal amplitude and prolonged signal decay produced by the PMCA4-GCaMP2 to the level similar with those produced by the PMCA4 ${ }^{\text {mut }} \mathrm{GCaMP} 2$.

GCaMP2 is the latest generation of genetically encoded calcium indicators (GECI) developed by J Nakai and colleagues $[13,28]$ based on the principle of circular permutation of fluorescent proteins. The GCaMP2 structure comprises of the 13-residue peptide of the $\mathrm{Ca}^{2+}$-calmodulin binding domain of the MLC kinase (M13), which is inserted upstream of the C-terminal fragment of EGFP. The N-terminal EGFP fragment with calmodulin is linked downstream of the above structure. Under basal conditions this recombinant protein produces a low fluorescent signal since the C-terminal fragment of EGFP precedes the N-terminal fragment. Binding with calcium will alter the conformation of GCaMP2 resulting in an increased fluorescent signal [14].

GCaMP2 has been used previously for calcium imaging in various models. Tallini et al. generated transgenic mice expressing GCaMP2 in the heart, which enabled the measurement of myocyte $\mathrm{Ca}^{2+}$ transients in vivo in all regions of the beating mouse heart [13]. In another study, Lee et al. linked GCaMP2 with the $\alpha 1$ and $\alpha 2$ subunits of the $\mathrm{Na}^{+} / \mathrm{K}^{+}$ ATPase pump and overexpressed these in astrocytes. These sensors were able to define a different subsarcolemmal calcium environment related to each subunit [12]. More recently, GCaMP2 was cloned to subcellular targeting proteins to target the GCaMP2 to certain sub-cellular localisations; an approach which proved successful in detecting subcellular local calcium. For example, the Lck-GCaMP2 was able to detect a sub-membrane calcium microdomain in neuronal cells [29], and the mito-GCaMP2 has been developed for mitochondrial calcium imaging [30]. Unlike other GCaMP2 sensors which were cloned to non-calciumregulating proteins, the PMCA4-GCaMP2 was the first trial to fuse this calcium sensor to a calcium pump.

Experiments using a specific inhibitor were needed to further confirm the findings. However, prior to this study there was no known small molecule which acts as a specific inhibitor of PMCA4 activity. Inhibitors used in previous studies have either been general inhibitors of the ATPase family such as lanthanum, vanadate and eosin [31-37], or general calmodulin antagonists such as calmidazolium [38]. Currently, carboxyeosin is the only available non-specific pharmacological inhibitor for PMCA [39]. However, carboxyeosin is a fluorescent compound and would interfere with the GCaMP2 signal. We therefore performed inhibitor screening and obtained a key result, i.e. the identification of aurintricarboxylic acid (ATA) as a potent PMCA4 inhibitor. ATA completely inhibited PMCA4 activity at a very low concentration $(1 \mu \mathrm{M})$. At higher concentrations (up to $100 \mu \mathrm{M}$ ) ATA has been reported to have several effects on different cell types. For example, ATA has been described as an anti-viral [40-45] and anti-apoptotic agent [51-53]. It also inhibits several types of nucleases (DNAse I, RNAse A, SI nuclease, exonuclease) [46-48]. In the present study we used ATA at a very low concentration and instantly detected the acute effects on the cells using a PMCA4-based fluorescent sensor. Therefore, the response detected by the GCaMP2 fusion molecules was very likely due to ATA action on PMCA4.

The PMCA4-GCaMP2 fusion protein may be very useful in further understanding the signalling interplay between PMCA4 and its interacting partners. Previously, we have described a physical interaction between PMCA4 and other signalling molecules in cardiomyocytes, notably nNOS, $\alpha 1$-syntrophin, calcineurin and RASSF1A [8,49-51]. It is known that overexpression of PMCA4 reduced the activity of its interacting partners, for example nNOS and calcineurin $[7,8,49]$. However, it is not known whether local calcium dynamics around PMCA4 are altered in the situation of excess or depleted expression of its interacting partners. PMCA4-GCaMP2 and PMCA4 ${ }^{\text {mut }}$ GCaMP2 can be used as novel tools to examine local calcium changes under these circumstances, in situ, which could offer insights into the role of these interactions during a number of cardiac disease processes.

The discovery of a specific pharmacological inhibitor for PMCA4 could be extremely useful, not only as a tool in the study of the pump's actions in the heart, but also in a number of other cell types and disease processes. Our studies in mice lacking PMCA4 [37] have found male mice to be infertile due to a defect in sperm motility, but otherwise males and females were both completely healthy under physiological conditions with a normal lifespan. Our findings were later confirmed by Okunade and colleagues who generated a similar PMCA4 knockout mouse model [52]. In addition a very recent GWAS study showed the PMCA4 gene to be associated with resistance to infection by malaria plasmodium in humans [53]. Therefore, our discovery that ATA is a specific inhibitor for PMCA4 could have further potential applications in the fields of contraception and anti-malaria treatment.

\section{Conclusion}

In this study we generated and analyzed the properties of two novel PMCA4 based calcium indicators, PMCA4-GCaMP2 and 
A

PMCA4-GCaMP2

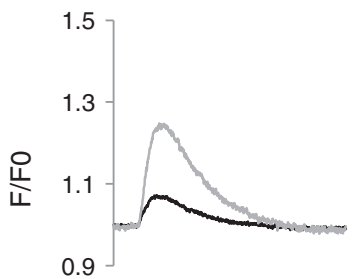

C

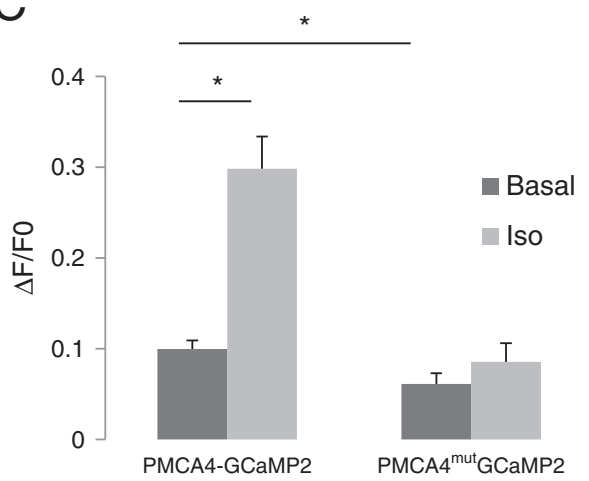

B
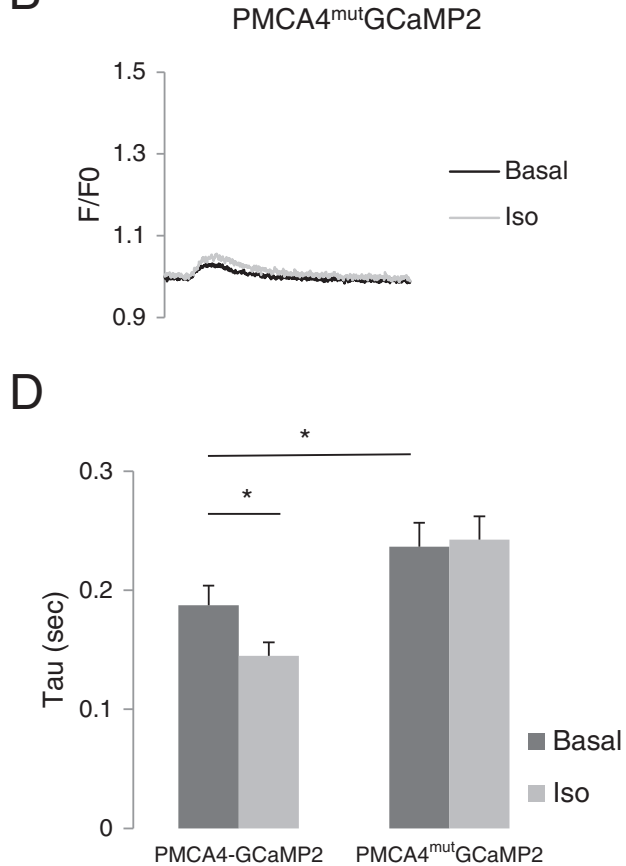

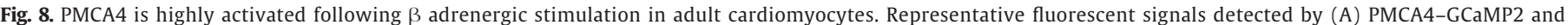

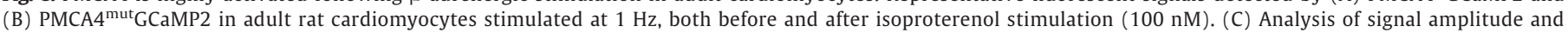

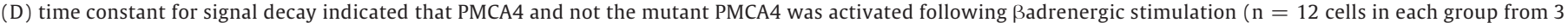
independent experiments, ${ }^{*} \mathrm{P}<0.05$ ).

PMCA $4{ }^{\text {mut }}$ GCaMP2. Together, PMCA4-GCaMP2 and PMCA4 ${ }^{\text {mut }}$ GCaMP2 can be used to monitor calcium dynamics around these fusion proteins. Those fusion molecules may also be useful in testing the efficacy of small molecules that can modulate PMCA4 function. In addition, using a similar approach, the GCaMP2 sensor could be useful for studying other calcium transporters such as SERCA, NCX, TRPCs and IP3 receptors during excitation-contraction coupling in cardiomyocytes. The second aspect of this study, our discovery of a specific PMCA4 inhibitor will enable further investigations into the precise role of PMCA4 in other cell types, with potential implications for the fields of fertility and malaria.

\section{Disclosure statement}

The authors have no conflict of interest to disclose.

\section{Acknowledgments}

We would like to thank Dr Junichi Nakai for providing the GCaMP2 construct. In addition, we would like to thank Dr. Michael I. Kotlikoff for his technical advice regarding the GCaMP2 imaging throughout the project. This work was supported by the Medical Research Council Programme and Research Grants to L.N. (G0500025 and G0802004) and by the NIHR Manchester Biomedical Research Centre. D.O. is supported by the British Heart Foundation Intermediate Fellowship (FS/09/046/28043).

\section{Appendix A. Supplementary data}

Supplementary data to this article can be found online at http://dx. doi.org/10.1016/j.yjmcc.2013.07.007.

\section{References}

[1] Kiriazis H, Kranias EG. Genetically engineered models with alterations in cardiac membrane calcium-handling proteins. Annu Rev Physiol 2000;62:321-51.
[2] Carafoli E, Stauffer T. The plasma membrane calcium pump: functional domains, regulation of the activity, and tissue specificity of isoform expression. J Neurobiol 1994;25:312-24

[3] Bers DM. Calcium fluxes involved in control of cardiac myocyte contraction. Circ Res 2000;87:275-81.

[4] Strehler EE, Zacharias DA. Role of alternative splicing in generating isoform diversity among plasma membrane calcium pumps. Physiol Rev 2001;81:21-50.

[5] Choi HS, Eisner DA. The effects of inhibition of the sarcolemmal Ca-ATPase on systolic calcium fluxes and intracellular calcium concentration in rat ventricular myocytes. Pflugers Arch 1999;437:966-71.

[6] Bers DM, Bassani JW, Bassani RA. Competition and redistribution among calcium transport systems in rabbit cardiac myocytes. Cardiovasc Res 1993;27: 1772-7.

[7] Mohamed TM, Oceandy D, Prehar S, Alatwi N, Hegab Z, Baudoin FM, et al. Specific role of neuronal nitric-oxide synthase when tethered to the plasma membrane calcium pump in regulating the beta-adrenergic signal in the myocardium. J Biol Chem 2009;284:12091-8.

[8] Schuh K, Uldrijan S, Telkamp M, Rothlein N, Neyses L. The plasmamembrane calmodulin-dependent calcium pump: a major regulator of nitric oxide synthase I. J Cell Biol 2001;155:201-5.

[9] Oceandy D, Cartwright EJ, Emerson M, Prehar S, Baudoin FM, Zi M, et al. Neuronal nitric oxide synthase signaling in the heart is regulated by the sarcolemmal calcium pump 4b. Circulation 2007;115:483-92.

[10] Wu X, Chang B, Blair NS, Sargent M, York AJ, Robbins J, et al. Plasma membrane Ca2 + -ATPase isoform 4 antagonizes cardiac hypertrophy in association with calcineurin inhibition in rodents. J Clin Invest 2009;119:976-85.

[11] Oceandy D, Mohamed TM, Cartwright EJ, Neyses L. Local signals with global impacts and clinical implications: lessons from the plasma membrane calcium pump (PMCA4). Biochim Biophys Acta 1813;2011:974-8.

[12] Lee MY, Song H, Nakai J, Ohkura M, Kotlikoff MI, Kinsey SP, et al. Local subplasma membrane Ca2 + signals detected by a tethered Ca2 + sensor. Proc Natl Acad Sci U S A 2006; 103:13232-7.

[13] Tallini YN, Ohkura M, Choi BR, Ji G, Imoto K, Doran R, et al. Imaging cellular signals in the heart in vivo: cardiac expression of the high-signal Ca2 + indicator GCaMP2. Proc Natl Acad Sci U S A 2006;103:4753-8.

[14] Kotlikoff MI. Genetically encoded Ca2 + indicators: using genetics and molecular design to understand complex physiology. J Physiol 2007:578:55-67.

[15] Zhou YY, Wang SQ, Zhu WZ, Chruscinski A, Kobilka BK, Ziman B, et al. Culture and adenoviral infection of adult mouse cardiac myocytes: methods for cellular genetic physiology. Am J Physiol 2000;279:H429-36.

[16] Oceandy D, Pickard A, Prehar S, Zi M, Mohamed TM, Stanley PJ, et al. Tumor suppressor ras-association domain family 1 isoform $A$ is a novel regulator of cardiac hypertrophy. Circulation 2009;120:607-16.

[17] Mohamed TM, Oceandy D, Zi M, Prehar S, Alatwi N, Wang Y, et al. Plasma membrane calcium pump (PMCA4)-neuronal nitric-oxide synthase complex regulates cardiac contractility through modulation of a compartmentalized cyclic nucleotide microdomain. J Biol Chem 2011;286:41520-9. 
[18] Mohamed TM, Baudoin-Stanley FM, Abou-Leisa R, Cartwright E, Neyses L, Oceandy D. Measurement of plasma membrane calcium-calmodulin-dependent ATPase (PMCA) activity. Methods Mol Biol 2010;637:333-42.

[19] Roach TI, Rebres RA, Fraser ID, Decamp DL, Lin KM, Sternweis PC, et al. Signaling and cross-talk by C5a and UDP in macrophages selectively use PLCbeta3 to regulate intracellular free calcium. J Biol Chem 2008;283:17351-61.

[20] Adamo HP, Filoteo AG, Enyedi A, Penniston JT. Mutants in the putative nucleotidebinding region of the plasma membrane $\mathrm{Ca}(2+)$-pump. A reduction in activity due to slow dephosphorylation. J Biol Chem 1995;270:30111-4.

[21] Hammes A, Oberdorf-Maass S, Rother T, Nething K, Gollnick F, Linz KW, et al. Overexpression of the sarcolemmal calcium pump in the myocardium of transgenic rats. Circ Res 1998;83:877-88.

[22] Fujimoto T. Calcium pump of the plasma membrane is localized in caveolae. J Cell Biol 1993;120:1147-57.

[23] Shigetomi E, Kracun S, Sofroniew MV, Khakh BS. A genetically targeted optical sensor to monitor calcium signals in astrocyte processes. Nat Neurosci 2010;13: 759-66.

[24] Tian L, Hires SA, Mao T, Huber D, Chiappe ME, Chalasani SH, et al. Imaging neural activity in worms, flies and mice with improved GCaMP calcium indicators. Nat Methods 2009;6:875-81.

[25] Monteith GR, Wanigasekara Y, Roufogalis BD. The plasma membrane calcium pump, its role and regulation: new complexities and possibilities. J Pharmacol Toxicol Methods 1998;40:183-90.

[26] James PH, Pruschy M, Vorherr TE, Penniston JT, Carafoli E. Primary structure of the cAMP-dependent phosphorylation site of the plasma membrane calcium pump. Biochemistry 1989;28:4253-8.

[27] Lohse MJ, Engelhardt S, Eschenhagen T. What is the role of beta-adrenergic signaling in heart failure? Circ Res 2003;93:896-906.

[28] Nakai J, Ohkura M, Imoto K. A high signal-to-noise $\mathrm{Ca}(2+)$ probe composed of a single green fluorescent protein. Nat Biotechnol 2001;19:137-41.

[29] Shigetomi E, Tong X, Kwan KY, Corey DP, Khakh BS. TRPA1 channels regulate astrocyte resting calcium and inhibitory synapse efficacy through GAT-3. Nat Neurosci 2012;15:70-80.

[30] Iguchi M, Kato M, Nakai J, Takeda T, Matsumoto-Ida M, Kita T, et al. Direct monitoring of mitochondrial calcium levels in cultured cardiac myocytes using a novel fluorescent indicator protein, GCaMP2-mt. Int I Cardiol 2012;158:225-34.

[31] Quist EE, Roufogalis BD. Determination of the stoichiometry of the calcium pump in human erythrocytes using lanthanum as a selective inhibitor. FEBS Lett 1975;50: 135-9.

[32] Sarkadi B, Szasz I, Gerloczy A, Gardos G. Transport parameters and stoichiometry of active calcium ion extrusion in intact human red cells. Biochim Biophys Acta 1977;464: 93-107.

[33] Cantley Jr LC, Josephson L, Warner R, Yanagisawa M, Lechene C, Guidotti G. Vanadate is a potent $(\mathrm{Na}, \mathrm{K})$-ATPase inhibitor found in ATP derived from muscle. J Biol Chem 1977;252:7421-3.

[34] Barrabin H, Garrahan PJ, Rega AF. Vanadate inhibition of the Ca2 + -ATPase from human red cell membranes. Biochim Biophys Acta 1980;600:796-804.

[35] Shmigol A, Eisner DA, Wray S. Carboxyeosin decreases the rate of decay of the $[\mathrm{Ca} 2+] \mathrm{i}$ transient in uterine smooth muscle cells isolated from pregnant rats. Pflugers Arch 1998;437:158-60.

[36] Kurnellas MP, Nicot A, Shull GE, Elkabes S. Plasma membrane calcium ATPase deficiency causes neuronal pathology in the spinal cord: a potential mechanism for neurodegeneration in multiple sclerosis and spinal cord injury. FASEB J 2005;19: 298-300.

[37] Schuh K, Cartwright EJ, Jankevics E, Bundschu K, Liebermann J, Williams JC, et al. Plasma membrane Ca2 + ATPase 4 is required for sperm motility and male fertility. J Biol Chem 2004;279:28220-6.

[38] Gietzen K, Sadorf I, Bader H. A model for the regulation of the calmodulin-dependent enzymes erythrocyte Ca2 + -transport ATPase and brain phosphodiesterase by activators and inhibitors. Biochem J 1982;207:541-8.

[39] Choi HS, Eisner DA. The role of sarcolemmal Ca2 + -ATPase in the regulation of resting calcium concentration in rat ventricular myocytes. J Physiol 1999;515(Pt 1):109-18.

[40] Hashem AM, Flaman AS, Farnsworth A, Brown EG, Van Domselaar G, He R, et al Aurintricarboxylic acid is a potent inhibitor of influenza A and B virus neuraminidases. PLoS One 2009; 4:e8350.

[41] Myskiw C, Deschambault Y, Jefferies K, He R, Cao J. Aurintricarboxylic acid inhibits the early stage of vaccinia virus replication by targeting both cellular and viral factors. J Virol 2007;81:3027-32.

[42] Chen Y, Bopda-Waffo A, Basu A, Krishnan R, Silberstein E, Taylor DR, et al. Characterization of aurintricarboxylic acid as a potent hepatitis $C$ virus replicase inhibitor. Antivir Chem Chemother 2009;20:19-36.

[43] Hung HC, Tseng CP, Yang JM, Ju YW, Tseng SN, Chen YF, et al. Aurintricarboxylic acid inhibits influenza virus neuraminidase. Antiviral Res 2009;81:123-31.

[44] He R, Adonov A, Traykova-Adonova M, Cao J, Cutts T, Grudesky E, et al. Potent and selective inhibition of SARS coronavirus replication by aurintricarboxylic acid. Biochem Biophys Res Commun 2004;320:1199-203.

[45] Hung HC, Chen TC, Fang MY, Yen KJ, Shih SR, Hsu JT, et al. Inhibition of enterovirus 71 replication and the viral 3D polymerase by aurintricarboxylic acid. J Antimicrob Chemother 2010;65:676-83.

[46] Hallick RB, Chelm BK, Gray PW, Orozco Jr EM. Use of aurintricarboxylic acid as an inhibitor of nucleases during nucleic acid isolation. Nucleic Acids Res 1977;4: 3055-64.

[47] Grollman AP, Stewart ML. Inhibition of the attachment of messenger ribonucleic acid to ribosomes. Proc Natl Acad Sci U S A 1968;61:719-25.

[48] Stewart ML, Grollman AP, Huang MT. Aurintricarboxylic acid: inhibitor of initiation of protein synthesis. Proc Natl Acad Sci U S A 1971;68:97-101.

[49] Buch MH, Pickard A, Rodriguez A, Gillies S, Maass AH, Emerson M, et al. The sarcolemmal calcium pump inhibits the calcineurin/nuclear factor of activated T-cell pathway via interaction with the calcineurin A catalytic subunit. J Biol Chem 2005;280:29479-87.

[50] Armesilla AL, Williams JC, Buch MH, Pickard A, Emerson M, Cartwright EJ, et al. Novel functional interaction between the plasma membrane Ca2 + pump $4 \mathrm{~b}$ and the proapoptotic tumor suppressor Ras-associated factor 1 (RASSF1). J Biol Chem 2004;279:31318-28.

[51] Williams JC, Armesilla AL, Mohamed TM, Hagarty CL, McIntyre FH, Schomburg S, et al. The sarcolemmal calcium pump, alpha-1 syntrophin and neuronal nitric oxide synthase are part of a macromolecular protein complex. J Biol Chem 2006;281:23341-8.

[52] Okunade GW, Miller ML, Pyne GJ, Sutliff RL, O'Connor KT, Neumann JC, et al Targeted ablation of plasma membrane Ca2 +-ATPase (PMCA) 1 and 4 indicates a major housekeeping function for PMCA1 and a critical role in hyperactivated sperm motility and male fertility for PMCA4. J Biol Chem 2004;279:33742-50.

[53] Timmann C, Thye T, Vens M, Evans J, May J, Ehmen C, et al. Genome-wide association study indicates two novel resistance loci for severe malaria. Nature 2012;489: 443-6. 